\title{
Bioinformatics analysis of gene expression profiles of dermatomyositis
}

\author{
LIANG-YUAN CHEN ${ }^{1}$, ZHAO-LEI CUI ${ }^{2}$, FAN-CUI HUA ${ }^{1}$, WENG-JING YANG $^{1}$, YE BAI ${ }^{1}$ and FENG-HUA LAN ${ }^{1}$ \\ ${ }^{1}$ Department of Clinical Genetics and Experimental Medicine, Fuzong Clinical School, Fujian Medical University, Fuzhou, \\ Fujian 350025; ${ }^{2}$ Department of Clinical Laboratory, Fujian Provincial Tumor Hospital, Fuzhou, Fujian 350014, P.R. China
}

Received August 15, 2015; Accepted August 17, 2016

DOI: $10.3892 / \mathrm{mmr} .2016 .5703$

\begin{abstract}
Dermatomyositis (DM) is a type of autoimmune inflammatory myopathy, which primarily affects the skin and muscle. The underlying mechanisms of DM remain poorly understood. The present study aimed to explore gene expression profile alterations, investigate the underlying mechanisms, and identify novel targets for DM. The GSE48280 dataset, which includes data from five DM and five normal muscle tissue samples, was obtained from the Gene Expression Omnibus. Firstly, differentially expressed genes (DEGs) were screened by limma package in R. Subsequently, functional and pathway enrichment analyses were performed using ClueGO from Cytoscape. Finally, protein-protein interaction (PPI) networks were constructed using STRING and Cytoscape, in order to identify hub genes. As a result, 180 upregulated and 21 downregulated genes were identified in the DM samples. The Gene Ontology enrichment analysis revealed that the type I interferon (IFN) signaling pathway was the most significantly enriched term within the DEGs. The Kyoto Encyclopedia of Genes and Genomes pathway analysis identified 27 significant pathways, the majority of which can be divided into the infectious diseases and immune system categories. Following construction of PPI networks, 24 hub genes were selected, all of which were associated with the type I IFN signaling pathway in DM. The findings of the present study indicated that type I IFNs may have a central role in the induction of DM. In addition, other DEGs, including chemokine (C-C motif) ligand 5, C-X-C motif chemokine 10, Toll-like receptor 3, DEXD/H-Box helicase 58, interferon induced with helicase $\mathrm{C}$ domain 1, interferon-stimulated gene 15 and MX dynamin-like GTPase 1, may be potential targets for DM diagnosis and treatment.
\end{abstract}

Correspondence to: Dr Feng-Hua Lan, Department of Clinical Genetics and Experimental Medicine, Fuzong Clinical School, Fujian Medical University, 156 Xier Huan Road, Gulou, Fuzhou, Fujian 350025, P.R. China

E-mail: fhlan2014dc@163.com

Key words: dermatomyositis, type I interferons, bioinformatics analysis, protein-protein interaction network, ClueGO

\section{Introduction}

Dermatomyositis (DM) is a type of idiopathic inflammatory myopathy (IM), which affects the skin, muscle and other organs, and is associated with significant morbidity and mortality (1). The incidence of DM is estimated at 9.63 cases per million individuals, according to a population-based study (2). DM affects children and adults, and occurs more commonly in female patients (3). DM is closely associated with an increased risk of malignancy (4), including ovarian, lung, pancreatic, stomach and colorectal cancers, and non-Hodgkin's lymphoma (5). The rarity of the disease, the different features of the multiple disease subsets, and the lethal complications associated with DM make it difficult to cure (6).

Several factors are thought to contribute to DM, including environmental factors and genetic susceptibility. The association between DM and various environmental triggers, including medication, sunlight and infection, have been examined (7). In addition, a genetic component may predispose individuals to DM; the most important genetic region associated with DM appears to be the major histocompatibility complex (MHC), and a few candidate genes, such as signal transducer and activator of transcription (STAT) 4 and tumor necrosis factor, have been reported to be relevant to the development of DM (8). The first case of DM was reported in 1875 (9); however, the underlying molecular mechanisms of this disease remain incompletely understood. Pathological studies of muscle tissue in DM have reported histological abnormalities in capillaries, and the infiltration of muscle by B cells, cluster of differentiation (CD) $4^{+} \mathrm{T}$ cells, macrophages and plasma cells, and perifascicular atrophy (10). Therefore, DM has classically been considered a humorally mediated autoimmune disease (10). Recently, a microarray study reported that type 1 interferon (IFN)-mediated immune pathogenesis is likely to be involved in DM (11). Identification of the involved molecular mechanisms may lead to the development of effective therapeutic approaches for the treatment of patients with DM.

The present study downloaded the GSE48280 dataset, and identified the differentially expressed genes (DEGs) between the DM and healthy control (HC) muscle tissue samples, in order to explore the molecular mechanisms underlying DM. In addition, functional and pathway enrichment analyses were conducted, and protein-protein interaction (PPI) networks of the DEGs were constructed to identify the key genes and 
signaling pathways in DM. The findings of the present study may be helpful to improve understanding regarding the pathogenesis of DM, and provide insight into the development of a novel therapeutic strategy.

\section{Data and methods}

Microarray data. The GSE48280 gene expression profile (12) was downloaded from the Gene Expression Omnibus database (http://www.ncbi.nlm.nih.gov/geo/query/acc. cgi?acc=GSE48280). The profile contained five patients with DM, five patients with polymyositis, four patients with inclusion body myositis and five $\mathrm{HCs}$, the muscle samples from which were analyzed based on the Affymetrix Human Gene 1.0 ST Array platform (Affymetrix, Santa Clara, CA, USA). In the present study, only DM and HC samples were analyzed via a series of bioinformatics methods.

Data preprocessing and differential expression analysis. The original CEL files were transformed to probe-level data. If several probes mapped to a single gene, the mean of the probes was used to determine the gene expression value. The probe-level data were converted to gene symbols using the Perl procedure (version 5.24.0; www.perl.org). Following normalization, the limma package (13) in $\mathrm{R}$ (version 3.2.2; www.r-project.org) was conducted to identify DEGs between the DM and HC samples. Only genes with a fold change (FC) value $(\log 2 \mathrm{FCl})>1$ and a $\mathrm{P}$-value $<0.05$ were regarded as DEGs, which were considered the signature genes of DM.

Gene Ontology (GO) and Kyoto Encyclopedia of Genes and Genomes (KEGG) pathway analyses. GO (geneontology.org) and KEGG (http://www.genome.jp/kegg/) pathway enrichment analyses for the identified DEGs were performed using Cytoscape software (version 3.2.1) with the ClueGO V2.1.7 plug-in (14). The ClueGO plug-in generates functionally grouped GO annotation networks for a large number of genes. GO categories were divided into biological process, molecular function, cellular component and immune system process terms. The P-value was calculated by right-sided hypergeometric tests, and Benjamini-Hochberg adjustment was used for multiple test correction. GO terms with a P-value $<0.01$, and KEGG pathways with a P-value $<0.05$ were considered significant.

PPI network construction and hub gene identification. The Search Tool for the Retrieval of Interacting Genes (STRING; http://string-db.org/) database is an online tool, which has been designed as pre-computed global resource to evaluate PPI information (15). In the present study, the STRING database was applied to construct PPI networks for the screened DEGs, with a combination score $>0.99$ as the threshold. Subsequently, the PPI networks were visualized and analyzed using Cytoscape software (version 3.2.1) (16) based on the STRING database.

According to a previous study regarding biological networks, the majority of PPI networks in the present study exhibited scale-free network properties (17). PPI networks have a small number of highly connected protein nodes (known as hubs) and many poorly connected nodes. The connectivity degree was statistically analyzed by their combined scores based on the database algorithms. The important nodes in the PPI networks were identified and labeled as hub genes. Since the majority of the PPI networks obeyed the scale-free attribution, node degree $>20$ was selected as the threshold to obtain hub genes in the present study.

\section{Results}

Identification of DEGs between DM and HC samples. According to the cut-off criteria of $\mathrm{P}<0.05$ and $\mid \log 2 \mathrm{FCl}>1.0$, 201 DEGs were identified between the five DM and five HC samples using the limma package. A total of 180 DEGs were upregulated and 21 DEGs were downregulated.

GO and KEGG pathway analyses. All DEGs were subjected to $\mathrm{GO}$ and KEGG analysis. Following $\mathrm{GO}$ enrichment analysis using ClueGO, a total of $143 \mathrm{GO}$ terms were associated with the DEGs. The top 20 significantly enriched GO terms are presented in Table I. The top five significantly enriched GO terms were as follows: Type I IFN signaling pathway, defense response, response to cytokine, cytokine-mediated signaling pathway, and cellular response to cytokine stimulus.

Following the KEGG analysis, 27 KEGG pathways that were significantly enriched with DEGs were identified (Table II). The top five significantly enriched KEGG pathways were as follows: Herpes simplex infection, influenza A, antigen processing and presentation, measles, and hepatitis $\mathrm{C}$.

PPI network construction and hub gene identification. The PPI networks were constructed using Cytoscape based on the STRING database, and included 71 nodes and 389 edges with a combined score $>0.99$ (Fig. 1). The degree of all nodes was analyzed and genes with a node degree $>20$ are listed in Table III. Genes with a high degree of association included: ISG15 ubiquitin-like modifier (ISG15), interferon regulatory factor 1 (IRF1), interferon-induced protein with tetratricopeptide repeats 1 (IFIT1), MHC, class I, A (HLA-A), HLA-B , HLA-C, guanylate binding protein 2 (GBP2), IRF9, 2'-5'-oligoadenylate synthetase-like (OASL), 2'-5'-oligoadenylate synthetase 1 (OAS1), OAS2, OAS3, STAT2, IFIT2, IFIT3, MX dynamin-like GTPasel (MX1), interferon induced transmembrane protein 1 (IFITM1), IFITM2, IFITM3, interferon, alpha-inducible protein 6 (IFI6), IFI27, IFI35, XIAP associated factor 1 (XAF1) and adenosine deaminase, RNA-specific (ADAR). Among those hub genes, ISG15 exhibited the highest degree (degree, 30).

\section{Discussion}

DM is an autoimmune disease, which seriously impacts the quality of life of affected patients. The underlying pathogenesis of DM remains elusive. The present study analyzed gene expression profiles, identified the DEGs associated with DM, and enabled the identification of potential therapeutic targets. In the present study, 201 DEGs were identified, of which 180 were upregulated and 21 were downregulated. Following GO functional enrichment analysis using ClueGO, the type I IFN signaling pathway was revealed to be the most significantly enriched GO term within the DEGs. Previous studies have reported that patients with DM exhibit an activated type I IFN 
Table I. Top 20 Gene Ontology (GO) functional enrichment analysis of differentially expressed genes $(\mathrm{P}<0.01)$.

\begin{tabular}{|c|c|c|c|}
\hline Category & Term & Count & P-value \\
\hline Immune system process & GO:0060337: Type I interferon signaling pathway & 31 & $3.45 \mathrm{E}-41$ \\
\hline Biological process & GO:0006952: Defense response & 84 & $1.34 \mathrm{E}-37$ \\
\hline Biological process & GO:0034097: Response to cytokine & 57 & $1.95 \mathrm{E}-37$ \\
\hline Biological process & GO:0019221: Cytokine-mediated signaling pathway & 47 & $5.39 \mathrm{E}-35$ \\
\hline Biological process & GO:0071345: Cellular response to cytokine stimulus & 49 & 3.39E-32 \\
\hline Biological process & GO:0009615: Response to virus & 38 & $3.46 \mathrm{E}-27$ \\
\hline Immune system process & GO:0034341: Response to interferon-gamma & 26 & $6.14 \mathrm{E}-26$ \\
\hline Biological process & GO:0051707: Response to other organism & 48 & $9.91 \mathrm{E}-25$ \\
\hline Biological process & GO:0098542: Defense response to other organism & 38 & $2.95 \mathrm{E}-23$ \\
\hline Immune system process & GO:0071346: Cellular response to interferon-gamma & 22 & $6.58 \mathrm{E}-22$ \\
\hline Immune system process & GO:0060333: Interferon-gamma-mediated signaling pathway & 19 & $3.28 \mathrm{E}-20$ \\
\hline Biological process & GO:0043901: Negative regulation of multi-organism process & 20 & $4.95 \mathrm{E}-19$ \\
\hline Biological process & GO:0019079: Viral genome replication & 17 & $1.89 \mathrm{E}-17$ \\
\hline Biological process & GO:0050792: Regulation of viral process & 20 & 4.17E-17 \\
\hline Biological process & GO:0043900: Regulation of multi-organism process & 29 & $1.63 \mathrm{E}-16$ \\
\hline Biological process & GO:0031347: Regulation of defense response & 37 & $1.86 \mathrm{E}-16$ \\
\hline Immune system process & GO:0045088: Regulation of innate immune response & 27 & $1.31 \mathrm{E}-15$ \\
\hline Immune system process & GO:0050778: Positive regulation of immune response & 36 & 4.79E-15 \\
\hline Biological process & GO:0044403: Symbiosis, encompassing mutualism through parasitism & 36 & $1.26 \mathrm{E}-14$ \\
\hline Biological process & GO:0035456: Response to interferon-beta & 9 & 8.91E-14 \\
\hline
\end{tabular}

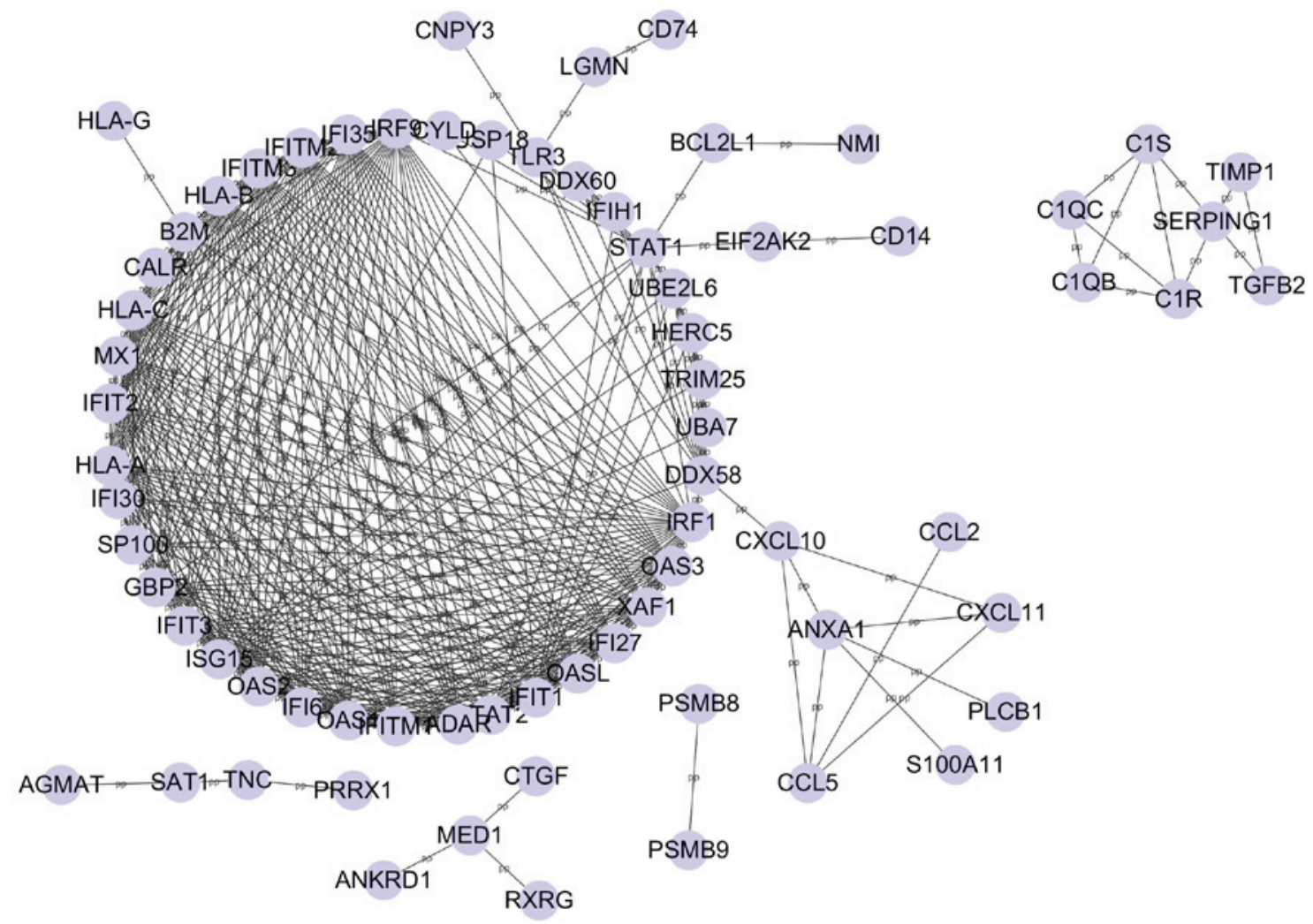

Figure 1. Constructed protein-protein interaction networks of differentially expressed genes. Nodes represent proteins, edges represent interactions between two proteins.

response, as demonstrated by increased type I IFN activity, and an IFN signature in the muscle, blood and skin $(1,18,19)$, which has also been described in patients with systemic lupus erythematosus, rheumatoid arthritis, Sjögren's syndrome and systemic 
Table II. Enriched Kyoto Encyclopedia of Genes and Genomes (KEGG) pathways of differentially expressed genes $(\mathrm{P}<0.05)$.

\begin{tabular}{|c|c|c|c|}
\hline Term & Description & Count & P-value \\
\hline KEGG:05168 & Herpes simplex infection & 21 & $2.31 \mathrm{E}-13$ \\
\hline KEGG:05164 & Influenza A & 18 & $7.66 \mathrm{E}-11$ \\
\hline KEGG:04612 & Antigen processing and presentation & 13 & $1.30 \mathrm{E}-10$ \\
\hline KEGG:05162 & Measles & 13 & $8.29 \mathrm{E}-08$ \\
\hline KEGG:05160 & Hepatitis C & 11 & 4.99E-06 \\
\hline KEGG:04610 & Complement and coagulation cascades & 8 & $1.14 \mathrm{E}-05$ \\
\hline KEGG:05133 & Pertussis & 8 & $1.85 \mathrm{E}-05$ \\
\hline KEGG:05150 & Staphylococcus aureus infection & 6 & $2.96 \mathrm{E}-04$ \\
\hline KEGG:05330 & Allograft rejection & 5 & 4.14E-04 \\
\hline KEGG:04145 & Phagosome & 9 & 4.41E-04 \\
\hline KEGG:05332 & Graft-versus-host disease & 5 & $5.44 \mathrm{E}-04$ \\
\hline KEGG:04940 & Type I diabetes mellitus & 5 & $6.20 \mathrm{E}-04$ \\
\hline KEGG:04622 & RIG-I-like receptor signaling pathway & 6 & $5.76 \mathrm{E}-04$ \\
\hline KEGG:05142 & Chagas disease (American trypanosomiasis) & 7 & 7.30E-04 \\
\hline KEGG:05320 & Autoimmune thyroid disease & 5 & $1.17 \mathrm{E}-03$ \\
\hline KEGG:05416 & Viral myocarditis & 5 & $1.79 \mathrm{E}-03$ \\
\hline KEGG:04620 & Toll-like receptor signaling pathway & 6 & 3.97E-03 \\
\hline KEGG:04623 & Cytosolic DNA-sensing pathway & 4 & $1.47 \mathrm{E}-02$ \\
\hline KEGG:05143 & African trypanosomiasis & 3 & $1.43 \mathrm{E}-02$ \\
\hline KEGG:04514 & Cell adhesion molecules (CAMs) & 6 & $1.55 \mathrm{E}-02$ \\
\hline KEGG:05020 & Prion diseases & 3 & $1.52 \mathrm{E}-02$ \\
\hline KEGG:05161 & Hepatitis B & 6 & $1.46 \mathrm{E}-02$ \\
\hline KEGG:04919 & Thyroid hormone signaling pathway & 5 & $2.32 \mathrm{E}-02$ \\
\hline KEGG:03050 & Proteasome & 3 & $2.29 \mathrm{E}-02$ \\
\hline KEGG:05144 & Malaria & 3 & $2.92 \mathrm{E}-02$ \\
\hline KEGG:04064 & NF-kappa B signaling pathway & 4 & $3.31 \mathrm{E}-02$ \\
\hline KEGG:00330 & Arginine and proline metabolism & 3 & 4.74E-02 \\
\hline
\end{tabular}

sclerosis (20). In addition, type 1 IFN-producing plasmacytoid dendritic cells are abundant in DM muscle and skin (21). When compared with other types of IM, the overproduction of IFN-I inducible transcripts and proteins in muscle tissue is a unique feature of DM (22). Furthermore, the iatrogenic administration of recombinant IFNs has been reported to induce DM (23). Taken together, these data indicated that the type I IFN signaling pathway may be important in the pathogenesis of DM.

A KEGG pathway enrichment analysis of the DEGs was conducted, and 27 significantly enriched pathways were identified. The majority of these pathways can be divided into two categories: Infectious diseases and the immune system. The enriched immune system-related pathways include antigen processing and presentation, complement and coagulation cascades pathway, RIG-I-like receptor (RLR) signaling pathway, Toll-like receptor (TLR) signaling pathway, and cytosolic DNA-sensing pathway. Among them, the TLR signaling, RLR signaling and cytosolic DNA-sensing pathways are responsible for detecting pathogens and generating innate immune responses. Type I IFNs are primarily induced by the activation of pattern-recognition receptors, such as TLRs, RLRs and cytoplasmic DNA sensor receptors (24). In the present study, chemokine (C-C motif) ligand 5 (CCL5), CD14, C-X-C motif chemokine 10 (CXCL10), CXCL11, STAT1 and TLR3
DEGs were enriched in the TLR signaling pathway, whereas CXCL10, CYLD lysine 63 deubiquitinase, DEXD/H-Box helicase 58 (DDX58), interferon induced with helicase $\mathrm{C}$ domain 1 (IFIH1), ISG15 and tripartite motif-containing protein 25 DEGs were enriched in the RLR signaling pathway. Furthermore, ADAR, CCL5, CXCL10 and DDX58 DEGs participated in the cytosolic DNA-sensing pathway. CCL5 is a key proinflammatory chemokine, which may have a role in driving recruitment of leukocytes, angiogenesis and fibrosis in chronic inflammatory diseases. Furthermore, CCL5 blockade is able to prevent immunopathology (25). CXCL10 and CXCL11 are members of the CXC subfamily of chemokines. Serum levels of CXCL10 are elevated in patients with active DM (26). CXCL10 has also been reported to be secreted by human skeletal muscle cells, which promote inflammation (27). A previous study using an animal model of C-protein-induced myositis demonstrated that suppressing CXCL10 with monoclonal antibodies was able to inhibit muscular inflammation (28). TLR3 is a member of the TLR family, which has a fundamental role in pathogen recognition and activation of innate immunity. An in vitro study revealed that TLR3 is involved in the overexpression of MHC-I in IM (29). Cappelletti et al reported that TLR3 is localized on vascular endothelial cells, muscle infiltrating cells and regenerating myofibers in DM (30). In addition, Li et al (31) 
Table III. Degree of each hub node in the protein-protein interaction networks.

\begin{tabular}{lc}
\hline Gene symbol & Degree \\
\hline ISG15 & 30 \\
IRF1 & 29 \\
IFIT1 & 28 \\
HLA-A, HLA-B, HLA-C, GBP2, IRF9 & 27 \\
OASL, OAS1, OAS2, OAS3 & 26 \\
STAT2 & 24 \\
IFIT2, IFIT3, MX1, IFITM1, IFITM2, IFITM3, & 23 \\
IFI6, IFI27, IFI35, XAF1, ADAR & \\
\hline & \\
ISG15, ISG15 ubiquitin-like modifier; IRF, interferon regulatory fac- \\
tor; IFIT, interferon-induced protein with tetratricopeptide repeats; \\
HLA, major histocompatibility complex, class I; GBP2, guanylate \\
binding protein 2; OASL, 2'-5'-oligoadenylate synthetase-like; OAS, \\
2'-5'-oligoadenylate synthetase; STAT2, signal transducer and activa- \\
tor of transcription 2; MX1, MX dynamin-like GTPase 1; IFITM, in- \\
terferon induced transmembrane protein 1; IFI, interferon, alpha-in- \\
$\begin{array}{l}\text { ducible protein; XAF1, XIAP associated factor 1; ADAR, adenosine } \\
\text { deaminase, RNA-specific. }\end{array}$
\end{tabular}

demonstrated that TLR-3 and RIG-I are implicated in the formation of perifascicular atrophy in DM.

RLRs comprise DDX58 (RIG-I), IFIH1 [also known as melanoma differentiation-associated protein 5 (MDA5)] and LGP2, and are known as cytosolic RNA receptors that induce the expression of type I IFN genes. Sato et al (32) detected anti-clinically amyopathic DM (CADM)-140 antibodies encoded by MDA5 in patients with amyopathic DM; of these patients, $50 \%$ had rapidly progressive interstitial lung disease (RP-ILD). In addition, it has been reported that anti-MDA5 autoantibodies may be useful for diagnosing DM, particularly the CADM and RP-ILD subsets among Asian cohorts (33). Suárez-Calvet et al (12) reported that RIG-I was upregulated in pathological muscle fibers from patients with DM compared with other types of IM, as determined by immunohistochemistry. In addition, human myotubes were shown to produce IFN- $\beta$ in response to RIG-I stimulation, and the autocrine effects of IFN- $\beta$ could further amplify the expression of IFN genes. The results of the present KEGG analysis highlighted the role of the type I IFN signaling pathway in the pathogenesis of DM, and suggested that DEGs associated with these pathways, including CCL5, CXCL10, TLR3, DDX58 and IFIH1, may be used as potential targets for DM diagnosis and treatment.

Hub nodes have more complex interactions compared with other genes, thus indicating that they have important roles in the underlying mechanisms of disease (34). Therefore, identification of hub genes may facilitate the development of effective therapeutic approaches for the treatment of patients with DM. The following DEGs: ISG15, IRF1, IFIT1, HLA-A, HLA-B, HLA-C, GBP2, IRF9, OASL, OAS1, OAS2, OAS3, STAT2, IFIT2, IFIT3, MX1, IFITM1, IFITM2, IFITM3, IFI6, IFI27, IFI35, XAF1 and ADAR were identified as hub genes in the PPI networks, thus indicating that these genes may have pivotal roles in DM development. Notably, all of these hub genes are involved in the type I IFN signaling pathway in DM. The
ISG15 gene had the highest degree in the PPI networks (degree, 30). Salajegheh et al demonstrated that ISG15 is one of the most upregulated genes in DM muscle compared with normal muscle, and the ISG15 protein localizes to atrophic myofibers, as determined by immunohistochemistry (23). ISG15 is a ubiquitin-like modifier (35), which is believed to function as a possible mediator of muscle atrophy via protein conjugation, and may contribute to disease activity in DM. MX1, IFIT1 and IFIT3 have been detected in DM muscle, and have previously been identified as ISG15-conjugated in IFN- $\beta$-stimulated HeLa cells (36). Furthermore, MX1 is an IFN-I-induced protein that provides innate defense against several viruses. A previous study reported that MX1 is abundant in DM muscle and skin, and is specifically expressed in DM but not in other types of IM (37). In addition, MX1 RNA in juvenile DM peripheral blood mononuclear cells is positively correlated with muscle involvement (38). Therefore, ISG15 and MX1 may contribute to disease activity, and may be considered potential molecular markers and effective treatment targets in DM.

Human leukocyte antigen (HLA-A, HLA-B, HLA-C) is the human-specific MHC. A previous study reported that MHC-I antigens were not detected in normal muscle, but were upregulated in IM muscle (39). In transgenic mice, overexpression of MHC-I is sufficient to induce myositis (40). The induction of MHC-I in IM may involve type I IFN and the 'IFN-I signature' is also induced in IMs (10). These data suggested that MHC-I may be considered a useful adjunctive diagnostic test for DM.

Other hub genes associated with the type I IFN signaling pathway may also have an important role in the pathogenesis of DM. For example, the OAS family proteins, which are predominantly induced by IFN type I, consist of OAS1, OAS2, OAS3 and OASL. OAS levels are strongly associated with autoimmune diseases (41). Sanayama et al (42) proposed that OASL may be a biomarker for the response of patients with rheumatoid arthritis to tocilizumab, which is the most commonly used drug to treat this disease. Identification of these genes and their precise roles may clarify the mechanism and offer useful information regarding the treatment of DM.

In conclusion, the present study attempted to explore the potential molecular mechanisms underlying DM using bioinformatics analyses. The findings of the present study may contribute to our understanding regarding the molecular mechanisms underlying DM. The identified DEGs, including CCL5, CXCL10, TLR3, DDX58, IFIH1, ISG15 and MX1, may potentially be used as targets for DM diagnosis and treatment. However, further studies are required to further verify this hypothesis.

\section{Acknowledgements}

This work was partly supported by the Natural Science Foundation of China (grant no. 81170562); The abstract of the current study was partly presented at the $14^{\text {th }}$ Chinese National Conference of Medical Genetics proceedings.

\section{References}

1. Wong D, Kea B, Pesich R, Higgs BW, Zhu W, Brown P, Yao Y and Fiorentino D: Interferon and biologic signatures in dermatomyositis skin: Specificity and heterogeneity across diseases. PLoS One 7: e29161, 2012. 
2. Bendewald MJ, Wetter DA, Li X and Davis MD: Incidence of dermatomyositis and clinically amyopathic dermatomyositis: A population-based study in Olmsted County, Minnesota. Arch Dermatol 146: 26-30, 2010.

3. Mantegazza R, Bernasconi P, Confalonieri P and Cornelio F. Inflammatory myopathies and systemic disorders: A review of immunopathogenetic mechanisms and clinical features. J Neurol 244: 277-287, 1997.

4. Nickoloff BJ and Nestle FO (eds): Dermatomyositis. In: Dermatologic Immunity. Curr Dir Autoimmun. Volume 10. Karger AG, Basel, pp313-332, 2008.

5. Hill CL, Zhang Y, Sigurgeirsson B, Pukkala E, Mellemkjaer L, Airio A, Evans SR and Felson DT: Frequency of specific cancer types in dermatomyositis and polymyositis: A population-based study. Lancet 357: 96-100, 2001

6. Antiga E, Kretz CC, Klembt R, Massi D, Ruland V, Stumpf C, Baroni G, Hartmann M, Hartschuh W, Volpi W, et al: Characterization of regulatory $\mathrm{T}$ cells in patients with dermatomyositis J Autoimmun 35: 342-350, 2010.

7. Reed AM and Ytterberg SR: Genetic and environmental risk factors for idiopathic inflammatory myopathies. Rheum Dis Clin North Am 28: 891-916, 2002.

8. Rothwell S, Cooper RG, Lamb JA and Chinoy H: Entering a new phase of immunogenetics in the idiopathic inflammatory myopathies. Curr Opin Rheumatol 25: 735-741, 2013.

9. Dalakas MC: Muscle biopsy findings in inflammatory myopathies. Rheum Dis Clin North Am 28: 779-798, 2002.

10. Hornung $\mathrm{T}$ and Wenzel J: Innate immune-response mechanisms in dermatomyositis: An update on pathogenesis, diagnosis and treatment. Drugs 74: 981-998, 2014.

11. Greenberg SA: Sustained autoimmune mechanisms in dermatomyositis. J Pathol 233: 215-216, 2014.

12. Suárez-Calvet X, Gallardo E, Nogales-Gadea G, Querol L, Navas M, Díaz-Manera J, Rojas-Garcia R and Illa I: Altered RIG-I/DDX58-mediated innate immunity in dermatomyositis. J Pathol 233: 258-268, 2014.

13. Diboun I, Wernisch L, Orengo CA and Koltzenburg M: Microarray analysis after RNA amplification can detect pronounced differences in gene expression using limma. BMC Genomics 7: 252, 2006

14. Bindea G, Mlecnik B, Hackl H, Charoentong P, Tosolini M, Kirilovsky A, Fridman WH, Pagès F, Trajanoski $Z$ and Galon $\mathrm{J}$ ClueGO: A Cytoscape plug-in to decipher functionally grouped gene ontology and pathway annotation networks. Bioinformatics 25: 1091-1093, 2009.

15. von Mering C, Huynen M, Jaeggi D, Schmidt S, Bork P and Snel B: STRING: A database of predicted functional associations between proteins. Nucleic Acids Res 31: 258-261, 2003.

16. Shannon P, Markiel A, Ozier O, Baliga NS, Wang JT, Ramage D, Amin N, Schwikowski B and Ideker T: Cytoscape: A software environment for integrated models of biomolecular interaction networks. Genome Res 13: 2498-2504, 2003

17. Lamb J, Crawford ED, Peck D, Modell JW, Blat IC, Wrobel MJ, Lerner J, Brunet JP, Subramanian A, Ross KN, et al: The Connectivity Map: Using gene-expression signatures to connect small molecules, genes, and disease. Science 313: 1929-1935, 2006.

18. Baechler EC, Bauer JW, Slattery CA, Ortmann WA, Espe KJ, Novitzke J, Ytterberg SR, Gregersen PK, Behrens TW and Reed AM: An interferon signature in the peripheral blood of dermatomyositis subjects is associated with disease activity. Mol Med 13: 59-68, 2007

19. Kao L, Chung L and Fiorentino DF: Pathogenesis of dermatomyositis: Role of cytokines and interferon. Curr Rheumatol Rep 13: 225-232, 2011.

20. Greenberg SA: A gene expression approach to study perturbed pathways in myositis. Curr Opin Rheumatol 19: 536-541, 2007.

21. Walsh RJ, Kong SW, Yao Y, Jallal B, Kiener PA, Pinkus JL, Beggs AH, Amato AA and Greenberg SA: Type I interferon-inducible gene expression in blood is present and reflects disease activity in dermatomyositis and polymyositis. Arthritis Rheum 56: 3784-3792, 2007.

22. Hall JC and Rosen A: Type I interferons: Crucial participants in disease amplification in autoimmunity. Nat Rev Rheumatol 6: 40-49, 2010

23. Salajegheh M, Kong SW, Pinkus JL, Walsh RJ, Liao A, Nazareno R, Amato AA, Krastins B, Morehouse C, Higgs BW, et al: Interferon-stimulated gene 15 (ISG15) conjugates proteins in dermatomyositis muscle with perifascicular atrophy. Ann Neurol 67: 53-63, 2010.
24. Härtlova A, Erttmann SF, Raffi FA, Schmalz AM, Resch U, Anugula S, Lienenklaus S, Nilsson LM, Kröger A, Nilsson JA, et al: DNA damage primes the type I interferon system via the cytosolic DNA sensor STING to promote anti-microbial innate immunity. Immunity 42: 332-343, 2015.

25. Marques RE, Guabiraba R, Russo RC and Teixeira MM Targeting CCL5 in inflammation. Expert Opin Ther Targets 17: 1439-1460, 2013.

26. Bilgic H, Ytterberg SR, Amin S, McNallan KT, Wilson JC, Koeuth T, Ellingson S, Newman B, Bauer JW, Peterson EJ, et al: Interleukin-6 and type I interferon-regulated genes and chemokines mark disease activity in dermatomyositis. Arthritis Rheum 60: 3436-3446, 2009.

27. Crescioli C, Sottili M, Bonini P, Cosmi L, Chiarugi P, Romagnani P, Vannelli GB, Colletti M, Isidori AM, Serio M, et al: Inflammatory response in human skeletal muscle cells: CXCL10 as a potential therapeutic target. Eur J Cell Biol 91: 139-149, 2012.

28. Kim J, Choi JY, Park SH, Yang SH, Park JA, Shin K, Lee EY, Kawachi H, Kohsaka $\mathrm{H}$ and Song YW: Therapeutic effect of anti-C-X-C motif chemokine 10 (CXCL10) antibody on C protein-induced myositis mouse. Arthritis Res Ther 16: R126, 2014.

29. Tournadre A, Lenief V, Eljaafari A and Miossec P: Immature muscle precursors are a source of interferon- $\beta$ in myositis: Role of Toll-like receptor 3 activation and contribution to HLA class I up-regulation. Arthritis Rheum 64: 533-541, 2012.

30. Cappelletti C, Baggi F, Zolezzi F, Biancolini D, Beretta O, Severa M, Coccia EM, Confalonieri P, Morandi L, Mora M, et al: Type I interferon and Toll-like receptor expression characterizes inflammatory myopathies. Neurology 76: 2079-2088, 2011.

31. Li L, Dai T, Lv J, Ji K, Liu J, Zhang B and Yan C: Role of Toll-like receptors and retinoic acid inducible gene $\mathrm{I}$ in endogenous production of type I interferon in dermatomyositis. J Neuroimmunol 285: 161-168, 2015.

32. Sato S, Hoshino K, Satoh T, Fujita T, Kawakami Y, Fujita T and Kuwana M: RNA helicase encoded by melanoma differentiation-associated gene 5 is a major autoantigen in patients with clinically amyopathic dermatomyositis: Association with rapidly progressive interstitial lung disease. Arthritis Rheum 60: 2193-2200, 2009.

33. Lu X, Peng Q and Wang G: Discovery of new biomarkers of idiopathic inflammatory myopathy. Clin Chim Acta 444: 117-125, 2015.

34. Langfelder P, Mischel PS and Horvath S: When is hub gene selection better than standard meta-analysis? PloS One 8: e61505, 2013.

35. D'Cunha J, Knight E Jr, Haas AL, Truitt RL and Borden EC: Immunoregulatory properties of ISG15, an interferon-induced cytokine. Proc Natl Acad Sci USA 93: 211-215, 1996.

36. Zhao C, Denison C, Huibregtse JM, Gygi S and Krug RM: Human ISG15 conjugation targets both IFN-induced and constitutively expressed proteins functioning in diverse cellular pathways. Proc Natl Acad Sci USA 102: 10200-10205, 2005.

37. Arshanapalli A, Shah M, Veerula V and Somani AK: The role of type I interferons and other cytokines in dermatomyositis. Cytokine 73: 319-325, 2015.

38. O'Connor KA, Abbott KA, Sabin B, Kuroda M and Pachman LM: MxA gene expression in juvenile dermatomyositis peripheral blood mononuclear cells: Association with muscle involvement. Clin Immunol 120: 319-325, 2006.

39. Salaroli R, Baldin E, Papa V, Rinaldi R, Tarantino L, De Giorgi LB, Fusconi M, Malavolta N, Meliconi R, D'Alessandro R and Cenacchi G: Validity of internal expression of the major histocompatibility complex class I in the diagnosis of inflammatory myopathies. J Clin Pathol 65: 14-19, 2012.

40. van der Pas J, Hengstman GJ, ter Laak HJ, Borm GF and van Engelen BG: Diagnostic value of MHC class I staining in idiopathic inflammatory myopathies. J Neurol Neurosurg Psychiatry 75: 136-139, 2004

41. Choi UY, Kang JS, Hwang YS and Kim YJ: Oligoadenylate synthase-like (OASL) proteins: Dual functions and associations with diseases. Exp Mol Med 47: e144, 2015.

42. Sanayama Y, Ikeda K, Saito Y, Kagami S, Yamagata M, Furuta S, Kashiwakuma D, Iwamoto I, Umibe T, Nawata Y, et al: Prediction of therapeutic responses to tocilizumab in patients with rheumatoid arthritis: Biomarkers identified by analysis of gene expressionin peripheral blood mononuclear cells using genome-wide DNA microarray. Arthritis Rheumatol 66: 1421-1431, 2014. 